\title{
Electron temperature and heat load measurements in the COMPASS divertor using the new system of probes
}

\author{
J. Adamek, ${ }^{1}$ J. Seidl, ${ }^{1}$ J. Horacek, ${ }^{1}$ M. Komm, ${ }^{1}$ T. Eich, ${ }^{2}$ R. Panek ${ }^{1}$, J. Cavalier, ${ }^{1,3}$ A. \\ Devitre, ${ }^{1,4}$ M. Peterka, ${ }^{1}$ P. Vondracek, ${ }^{1,5}$ J. Stöckel, ${ }^{1}$ D. Sestak, ${ }^{1}$ O. Grover, ${ }^{1,6}$ P. \\ Bilkova, ${ }^{1}$ P. Böhm, ${ }^{1}$ J. Varju, ${ }^{1}$ A. Havranek, ${ }^{1,7}$ V. Weinzettl, ${ }^{1}$ J. Lovell, ${ }^{8,9}$ M. Dimitrova, ${ }^{1}$ K. \\ Mitosinkova, ${ }^{1,5}$ R. Dejarnac, ${ }^{1}$ M. Hron, ${ }^{1}$ and the COMPASS team and the EUROfusion \\ MST1 team ${ }^{10}$ \\ ${ }^{1}$ Institute of Plasma Physics of the CAS, Prague, Czech Republic \\ ${ }^{2}$ Max-Planck-Institut für Plasmaphysik, Garching, Germany \\ ${ }^{3}$ Institut Jean Lamour IJL, Université de Lorraine, Vandouvre-lès-Nancy, France \\ ${ }^{4}$ School of Physics, University of Costa Rica, San José, Costa Rica \\ ${ }^{5}$ Faculty of Mathematics and Physics, Charles University, Prague, Czech Republic \\ ${ }^{6}$ Faculty of Nuclear Physics and Engineering, Czech Technical University in Prague, Prague, Czech Republic \\ ${ }^{7}$ Faculty of Electrical Engineering, Czech Technical University in Prague, Prague, Czech Republic \\ ${ }^{8}$ Centre for Advanced Instrumentation, Department of Physics, Durham University, Durham, United Kingdom \\ ${ }^{9}$ Culham Centre for Fusion Energy, Culham Science Centre, Abingdon, Oxon, United Kingdom \\ ${ }^{10}$ See the author list of "Overview of progress in European Medium Sized Tokamaks towards an integrated plasma- \\ edge/wall solution" by H. Meyer et al., to be published in Nuclear Fusion Special issue: Overview and Summary Reports \\ from the 26th Fusion Energy Conference (Kyoto, Japan, 17-22 October 2016)
}

E-mail: adamek@ipp.cas.cz

Keywords: COMPASS, divertor, heat load, ELM, electron temperature, Ball-pen probe, Langmuir probe.

\begin{abstract}
A new system of probes was recently installed in the divertor of tokamak COMPASS in order to investigate the ELM energy density with high spatial and temporal resolution. The new system consists of two arrays of rooftop-shaped Langmuir probes (LPs) used to measure the floating potential or the ion saturation current density and one array of Ball-pen probes (BPPs) used to measure the plasma potential with a spatial resolution of $\sim 3.5 \mathrm{~mm}$. The combination of floating BPPs and LPs yields the electron temperature with microsecond temporal resolution. We report on the design of the new divertor probe arrays and first results of electron temperature profile measurements in ELMy Hmode and L-mode. We also present comparative measurements of the parallel heat flux using the new probe arrays and fast infrared termography (IR) data during L-mode with excellent agreement between both techniques using a heat power transmission coefficient $\gamma=7$. The ELM energy density $\varepsilon_{\|}$was measured during a set of NBI assisted ELMy H-mode discharges. The peak values of $\varepsilon_{\|}$were compared with those predicted by model and with experimental data from JET, AUG and MAST with a good agreement.
\end{abstract}




\section{INTRODUCTION}

Prediction of a heat flux incident on the plasma facing components of the ITER tokamak during ELMy H-mode is a key issue to avoid the risk of their thermal damage. The investigation of the ELM and inter-ELM parallel heat flux on the divertor is nowadays routinely performed using infrared termography (IR) in different fusion devices like JET and AUG $[1,2,3]$ and scaled towards the ITER tokamak. These measurements are also used as an input for modeling studying the risk of melting of different ITER divertor components [4]. The ELMs and their filamentary structure can be also investigated in the divertor region by probe techniques. The Langmuir probe (LP) has been implemented in most fusion experiments, in particular at the boundary $[5,6,7]$ and in the divertor region $[8,9,10]$ of tokamaks, as it can provide high-resolution direct measurements of several plasma parameters. In the COMPASS tokamak, 39 standard LPs are routinely operated $[11,12]$ to measure either the floating potential or the ion saturation current with high temporal resolution. Each probe can also provide the electron temperature by the swept probe technique, but at low temporal resolution $\sim 1 \mathrm{kHz}$ [13]. Sweeping can be performed to investigate ELMs via fitted conditionally-averaged $I-V$ characteristics [14], but this approach may underestimate the resulting ELM peak values of the electron temperature and parallel heat flux [7]. Resolving fast transients such as the ELMs and their filamentary structure requires electron temperature measurements of high temporal resolution (in the order of $\sim 1 \mu$ s), which can be achieved by combined probe techniques such as triple probes [15, 16, 17], mirror probes [18] or Ballpen probes [7]. The Ball-pen probe (BPP) is mainly used to measure the plasma potential in the Scrape Off Layer (SOL) $[19,20,21]$. The combination of a BPP potential $\left(\Phi^{\mathrm{BPP}}\right)$ and a LP potential $\left(V_{\mathrm{fl}}\right)$ and the equation

$$
T_{\mathrm{e}}=\left(\Phi^{B P P}-V_{\mathrm{fl}}\right) / \alpha
$$

yields the electron temperature with microsecond time resolution [22, 23], as was recently studied by 3D Particle-InCell simulations [24]. On the COMPASS tokamak, a new system of probes has been recently installed in the divertor region to systematically investigate the electron temperature and parallel heat flux during L-modes and ELMy Hmodes with high temporal $(\sim 1 \mu \mathrm{s})$ and good spatial $(\sim 3.5 \mathrm{~mm})$ resolution. In the following section, we report on the design of the new system of divertor probes. Section III explains the calibration of the BPP/LP technique. First measurements of the electron temperature in the divertor region of COMPASS during L-mode and ELMy H-mode discharges are presented in Section IV, where we also provide an example of the time evolution of the electron 
temperature near the outer strike point during density ramp-up from the first COMPASS detachment experiments. Probe measurements of parallel heat flux are then benchmarked against infrared data in an L-mode discharge. Finally, the ELM energy density profiles and peak ELM energy density values measured on COMPASS using probes are compared to the scaling predictions from JET, AUG and MAST infrared data [25].

\section{DESIGN OF NEW DIVERTOR BALL-PEN AND LANGMUIR PROBES}

The new diagnostic system consists of two arrays of rooftop-shaped LPs (labelled LPA and LPB, comprising $2 \times 55$ probes minus $2 \times 2$ missing LPs in the private flux region) and one array of BPPs (labelled BPP, comprising 56 probes) as can be seen in Fig. 1. The concept is based on the results from a previous design implemented in the

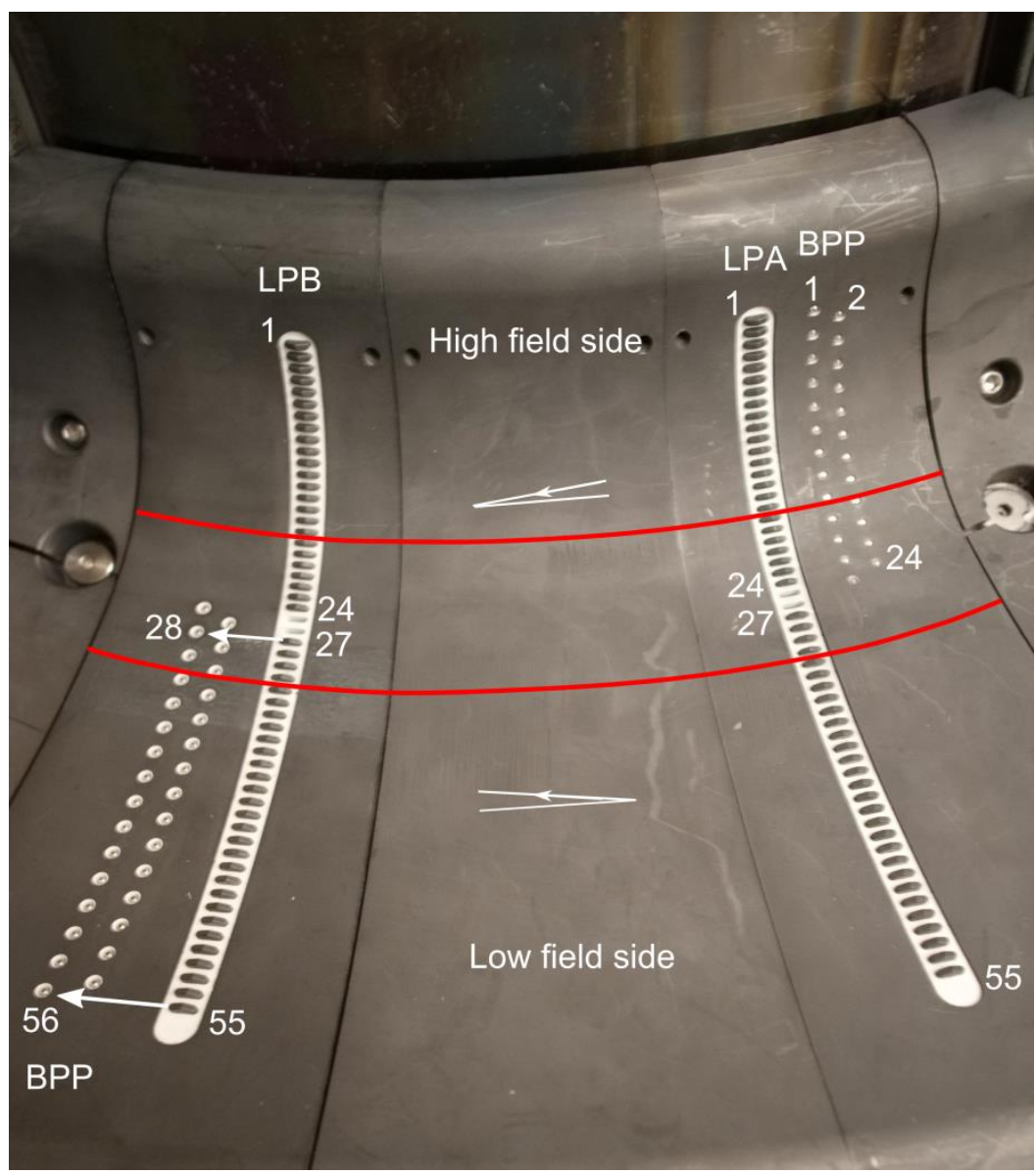

Fig. 1: Photo of the Ball-pen probe (BPP) and the rooftop-shaped Langmuir probe arrays (LPA and LPB) mounted on the divertor targets of COMPASS. The poloidal distance between neighboring probes is $\sim 3.5 \mathrm{~mm}$. The red lines show the approximate position of the strike points during the flattop of L-mode discharge \#13025 $\left(B_{\mathrm{T}}=1.15 \mathrm{~T}, I_{\mathrm{P}}=220 \mathrm{kA}\right.$, $\left.n_{\mathrm{e}}=4 \cdot 10^{19} \mathrm{~m}^{-3}\right)$. It also shows the magnetic field line orientation for a standard plasma current and toroidal magnetic field orientation. 
COMPASS divertor in 2014 [7]. The new BPPs are made of a boron nitride shielding tube, 3 mm in diameter, and a stainless-steel collector, $2 \mathrm{~mm}$ in diameter. The BPP collectors are retracted to a depth $\mathrm{h} \sim 0.4 \mathrm{~mm}$ within the shielding tube and provide plasma potential measurements. A schematic of the rooftop-shaped LP is shown in Fig.2. The probes are made of graphite protruding $1.5 \mathrm{~mm}$ into the plasma and provide floating potential or ion saturation current measurements. Both LP arrays are fixed in a boron-nitride holder, which is retracted $1 \mathrm{~mm}$ below the graphite surface. The rooftop-shaped LPs have a 20-degree chamfer with a total exposed area above the divertor target of $S_{\mathrm{LP}}=22 \mathrm{~mm}^{2}$ and projected area (on one side) of $S_{\mathrm{LP} \perp}=2.8 \mathrm{~mm}^{2}$ to reduce the impact of parallel heat flux and avoid the risk of a self-emitting regime. The design of LPs partially follows the previous design of standard divertor Langmuir probes operating on COMPASS [11]. Similar rooftop-shaped LPs are also used in the divertor of the DIII-D tokamak [8] and on the ASDEX Upgrade high heat flux probe head [6]. Some other tokamaks use the flush mounted LP design $[6,26]$ as it strongly reduces the impact of parallel heat flux on the probe surface without disturbing the plasma along the magnetic field lines. A drawback of flush mounted LPs is that they are sensitive to sheath-expansion effects [27] and its characteristics ( $I-V$ curve and projected area) strongly depend on the magnetic field line inclination [28].

The BPPs on the new divertor array are implemented directly on the divertor target as flush mounted probes and therefore the BPPs can measure the plasma potential in the vicinity of each corresponding LP, providing local measurements of the electron temperature. It is approximately toroidal distance of $2 \mathrm{~cm}$ between neighboring BPP and LP in Fig. 1. It should be noted that the inclination of the magnetic field lines changes sign within the private flux region, thus the BPPs must be placed behind the LPs on the Low Field Side (LFS) and in front of the LPs on the High Field Side (HFS) with respect to the magnetic field line orientation, also shown in Fig. 1. The absolute value of the magnetic field line inclination angle is approximately 1 degree on the strike points and increases towards 3 degrees far into the SOL. A sufficiently large toroidal distance, found by field line tracing

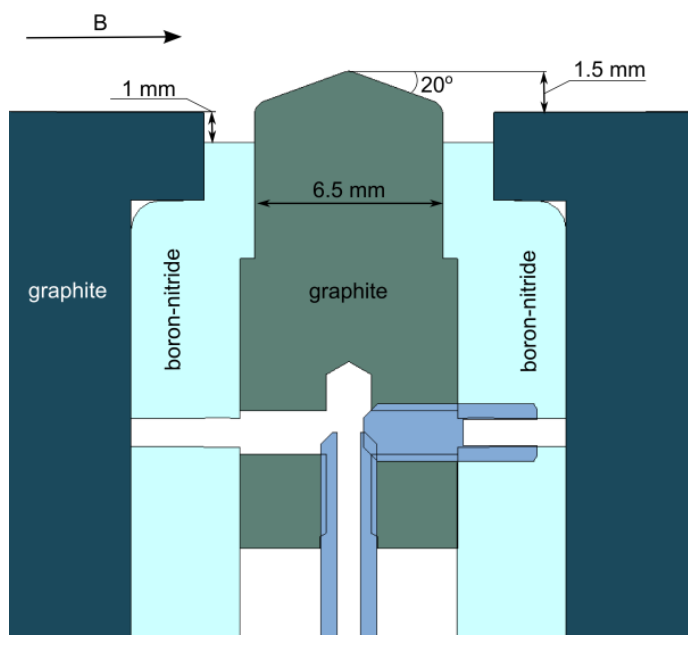

Fig. 2: Cross-section schematic of a COMPASS divertor rooftop-shaped Langmuir probe. The probe collector, made of graphite, is fixed inside a boron-nitride holder. 
calculations, was left between the two LP arrays to avoid mutual shadowing near the strike points and in the SOL. The mutual shadowing could not be avoided inside the private flux region were the field line inclination is close to zero.

The system of divertor probes is connected to dedicated electronics consisting of 200 independent channels for potential or current measurements. Each channel directly monitors the floating potential, the ion saturation current with variable biasing voltage $V_{\text {bias }}=\{-200 \mathrm{~V},-270 \mathrm{~V}\}$ (internal power supply) or the $I-V$ characteristic of a BPP or a LP (external power supply). Each channel consists of a potential or current measuring circuit and a small power supply based on a capacitor, making each channel independent. The capacity of the capacitors is sufficient to provide continuous measurements during a typical COMPASS discharge $(\mathrm{t}<0.3 \mathrm{~s})$ with a current limitation of 2 A per channel. All probe signals are acquired by the D-TACQ electronics with a sampling frequency $f=4 \mathrm{MSa} \cdot \mathrm{s}^{-1}$.

\section{CHARACTERISTICS OF ROOFTOP-SHAPED LANGMUIR PROBES}

Previous experiments on COMPASS, ASDEX Upgrade, MAST and ISTTOK [7, 22, 23], have shown that the combination of a BPP and a LP can provide the value of the electron temperature. Investigation of the electron temperature, $T_{\mathrm{e}}$, with microsecond temporal resolution is based on the fast potential measurements provided by neighboring BPPs and LPs using the equation (1), which were already presented in [7, 22, 29, 30]. The coefficient $\alpha$ is given by the characteristics of the LP and the BPP as $\alpha=\alpha_{\mathrm{LP}}-\alpha_{\mathrm{BPP}}$. The coefficients $\alpha_{\mathrm{LP}}$ and $\alpha_{\mathrm{BPP}}$ are equal to the logarithm of the ratio $\mathscr{R}=I_{\text {sat_e }} / I_{\text {sat_i }}$ of the electron and ion saturation currents obtained from the LP and BPP $I-V$ characteristics, as discussed in [7, 22, 24]. We have found that the characteristics of the new divertor BPPs are similar to those of the BPPs implemented by the old COMPASS divertor probe array [7]. We thus keep the same coefficient $\alpha_{\mathrm{BPP}}=0.6$ for deuterium plasmas. It must be noted that the current in the $I-V$ characteristic of the BPPs located in the far SOL on the HFS is strongly suppressed. This is probably due to a shadow from the unintended tilting of the new divertor tiles with respect to the toroidal curvature of the magnetic flux surfaces at this location. This issue has not been fully explored yet and we therefore restrict our profile measurements to the LFS.

The design of rooftop-shaped LPs significantly differs from the more common cylindrical Langmuir pins, also used on COMPASS, ASDEX Upgrade and ISTTOK [22, 21, 30]. The rooftop-shaped LPs significantly reduce (down to 20 degrees) the incidence angle of the magnetic field lines with respect to the probe surface, which 
affects the shape of the $I-V$ characteristics and the resulting value of $\mathscr{R}$, as shown in [28]. We have performed systematic measurements of LP $I-V$ characteristics at different locations of the divertor to determine the ratio $\mathfrak{R}$ and the coefficient $\alpha_{\mathrm{LP}}$. The measurements were performed during L-mode discharges on a shot-to-shot basis. In doing so, we have applied a general 4-parameter fitting function [31, 32], which takes into account the non-saturation of the
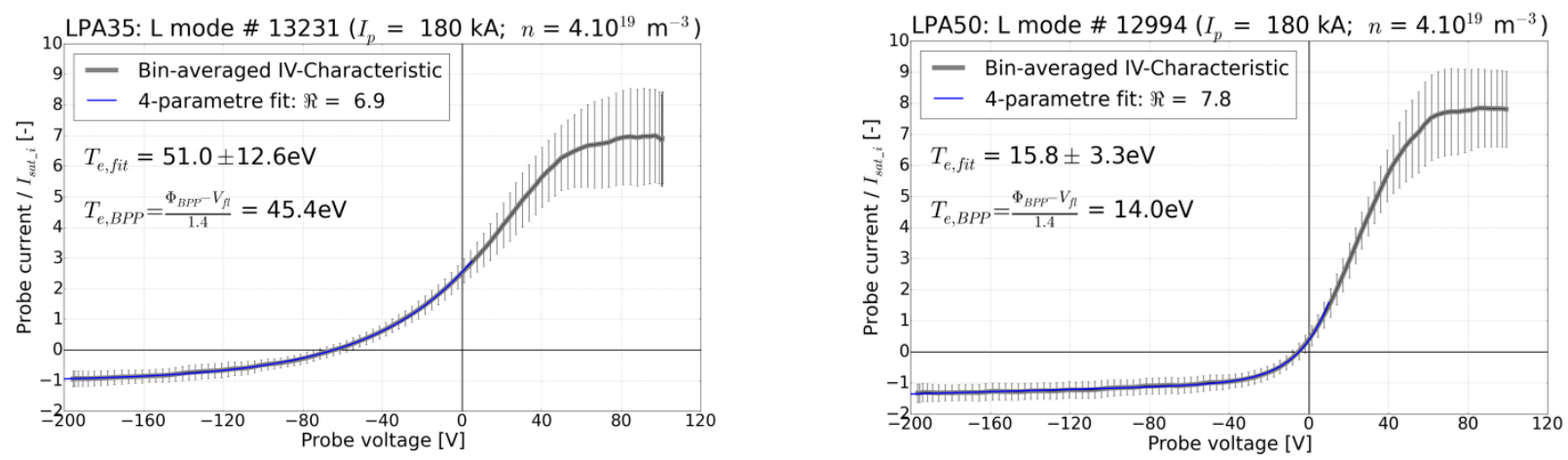

Fig. 3: Sample $I-V$ characteristics of probes LPA35 (left) and LPA50 (right) both located on the LFS, in the vicinity of the strike point and far into the SOL, respectively. The $I$ - $V$ characteristics are obtained during the whole flattop of similar L-mode discharges. Both $I-V$ characteristics are normalized to the ion saturation current. The saturated value of the electron branch thus yields the ratio $\Re$.

ion current, to obtain the electron temperature $T_{\mathrm{e}, \mathrm{fit}}$, the floating potential, the ion saturation current, $I_{\text {sat } \_\mathrm{i}}$, and the linear coefficient describing the non-saturated ion branch of the $I$ - $V$ characteristic. Sample $I$ - $V$ characteristics of probes LPA35 and LPA50, that are located on the LFS, in the vicinity of strike point and far into the SOL, respectively, are shown in Fig. 3. Both $I-V$ characteristics are obtained during the flat-top of similar L-mode discharges and are normalized to the value of $I_{\text {sat_i }}$. It can be seen in Fig. 3 that both characteristics are well saturated in the electron branch, defining a ratio $R=I_{\text {sat_e }} / I_{\text {sat_i }}$. By investigation of the $I-V$ characteristics of the LPs, we have found the value of $\Re$ to be similar at different locations on the divertor LFS and HFS, except in the far-SOL on the HFS and deep into the private flux region. We thus calculated a statistical value of $\Re=7.1 \pm 0.7$ which yields the coefficient $\alpha_{\mathrm{LP}}=\ln (\Re)=2.0 \pm 0.1$. The averaged value $\Re \sim 7.1$ is close to the value extrapolated from [28] for a 20degree chamfer, $\Re \sim 8.5$. We subsequently obtained an averaged value of the coefficient $\alpha=\alpha_{\mathrm{LP}}-\alpha_{\mathrm{BPP}}=1.4$ for deuterium plasmas allowing the electron temperature $T_{\mathrm{e}}$ to be calculated by $T_{\mathrm{e}}=\left(\Phi^{\mathrm{BPP}}-V_{\mathrm{fl}}\right) / 1.4$. For LPA35 and LPA50, Fig. 3 also compares the values of $T_{\mathrm{e}, \text { fit }}$ obtained by 4-parameter fitting to those given by the difference of floating potentials $\Phi^{\mathrm{BPP}}$ and $V_{\mathrm{fl}}$ of BPP and LPB, $T_{\mathrm{e}, \mathrm{BPP}}$, respectively, located on the same poloidal positions (see the divertor probes in Fig. 1). We observe that both techniques yield similar values within the error bar. It should be noted that the value of the ratio $\mathfrak{R}$ of roof-shaped LPs located in the far-SOL on the HFS is higher, probably due to 
the same shadowing observed on the BPP characteristics at this location. The profile measurements in the next section will therefore be focused on the LFS of the divertor.

\section{PROFILE MEASUREMENTS IN L-MODE AND ELMy H-MODE DISCHARGES}

Profile measurements of the electron temperature on the outer divertor target were performed during L-mode and NBI assisted ELMy H-mode discharges; an example is plotted in Fig 4, where all profiles are mapped to the midplane and fitted using the "Eich function" [2] i.e. exponential decay described by decay length $\lambda$ in the radial direction, convoluted with a Gaussian function with thickness $S$. The value $S$ represents plasma diffusion into the private flux region driven by strong pressure gradient. The L-mode $T_{\mathrm{e}}$ profile is obtained during the whole flattop of discharge \#13021. The profile has a peak value $T_{\mathrm{e} \_ \text {max }} \sim 40 \mathrm{eV}$ with an e-folding length $\lambda_{T e} \sim 4 \mathrm{~mm}$. The ELM peak

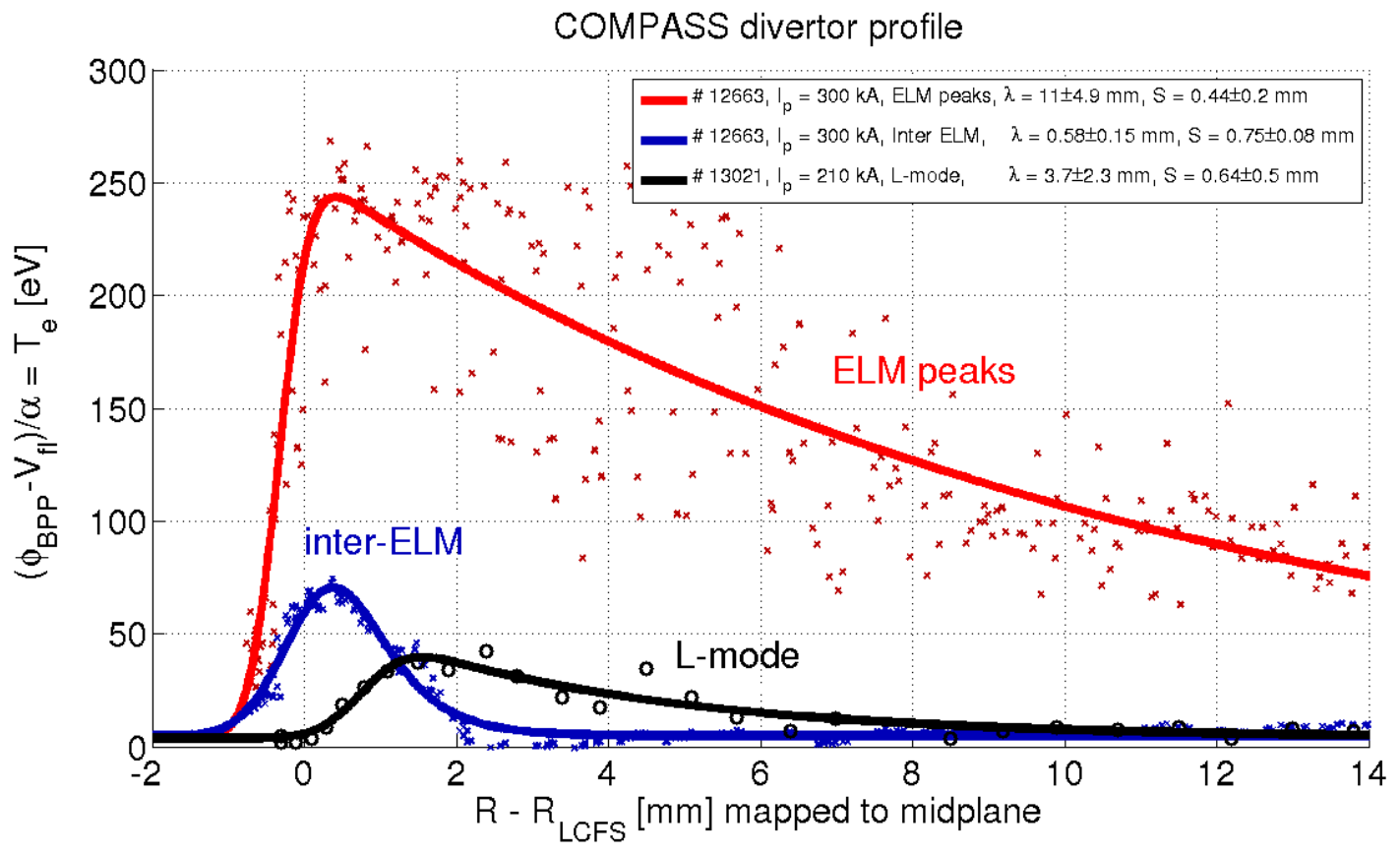

Fig. 4: Electron temperature profiles obtained on the LFS of the COMPASS divertor region during NBI assisted ELMy H-mode discharge $\# 12663\left(P_{\mathrm{NBI}}=220 \mathrm{~kW}, I_{\mathrm{P}}=300 \mathrm{kA}, n_{\mathrm{e}}=7-8 \cdot 10^{19} \mathrm{~m}^{-3}\right)$ with ELM peaks and inter-ELM values; and L-mode discharge \#13021 $\left(I_{\mathrm{P}}=210 \mathrm{kA}, n_{\mathrm{e}}=5 \cdot 10^{19} \mathrm{~m}^{-3}\right)$. All profiles are mapped to the outer midplane. The ELM peaks (red) and inter-ELM (blue) profiles include data of 10 Type-I ELMs. The L-mode profile (black) is obtained during the flattop phase.

data were collected during 10 Type-I ELMs [33] and the crosses represent $T_{\mathrm{e}}$ maxima appearing at each radius during a single ELM. For the ELM peaks the Eich fit gives $T_{\mathrm{e} \_ \text {max }} \sim 240 \mathrm{eV}$ with $\lambda_{T e} \sim 11.0$ mm, which represent a significant broadening of the e-folding length with respect to the L-mode profile. The maximum value of the electron temperature $T_{\mathrm{e}_{-} \max } \sim 240 \mathrm{eV}$ is close to the average value of the pedestal electron temperature $T_{\mathrm{e} \text {,ped }} \sim 250 \mathrm{eV}$ 

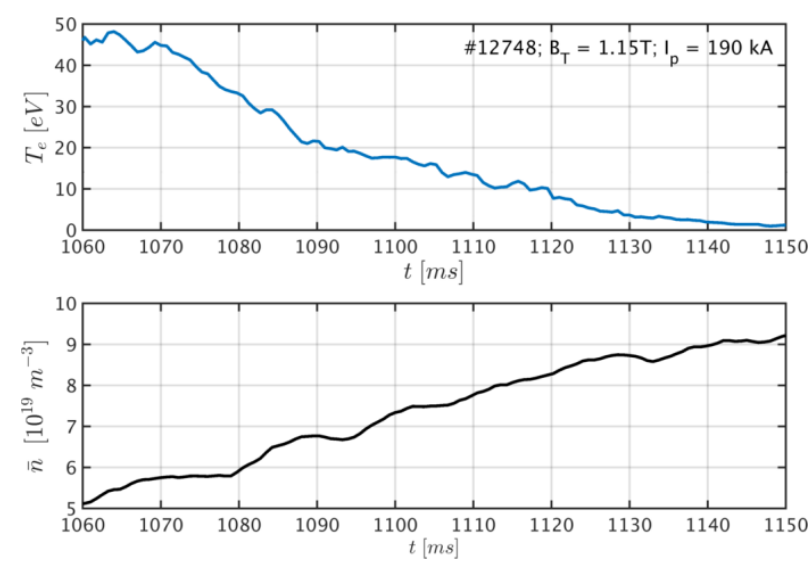

Fig. 5: Temporal evolution of the electron temperature in the vicinity of the LFS strike point (top) and line-averaged density (bottom) during density ramp-up in L-mode discharge \#12748 $\left(I_{\mathrm{P}}=\right.$ $\left.220 \mathrm{kA}, n_{\mathrm{e}}=4-9 \cdot 10^{19} \mathrm{~m}^{-3}\right)$. The values of the electron temperature are smoothed over $5 \mathrm{~ms}$ (moving average) to remove the fluctuations.

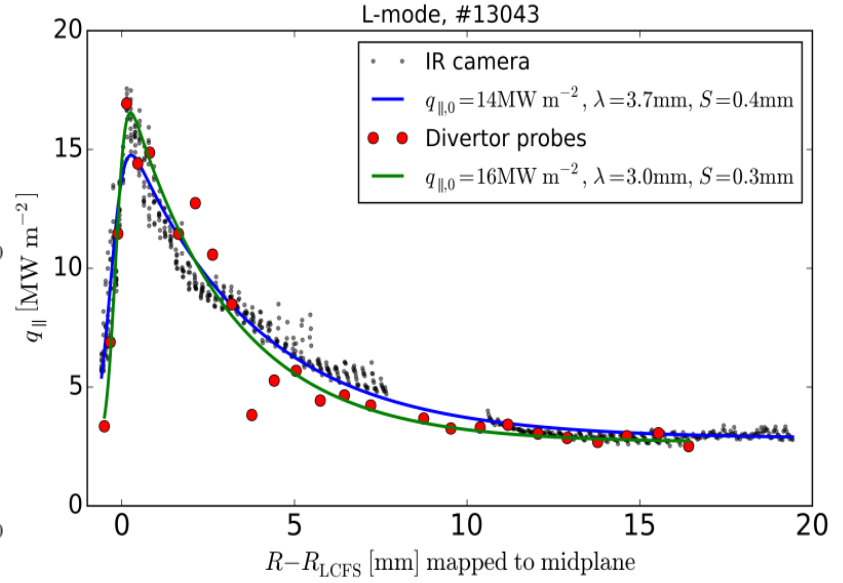

Fig. 6: Comparison of the parallel heat flux profiles on the divertor LFS as measured by divertor probes and IR camera during L-mode discharge \# $13043\left(I_{P}=240\right.$ $\left.\mathrm{kA}, n_{\mathrm{e}}=4 \cdot 10^{19} \mathrm{~m}^{-3}\right)$. Both profiles are mapped to the outer midplane and fitted by the "Eich function" [2].

obtained from the COMPASS pedestal scaling for $P_{\mathrm{NBI}}=220 \mathrm{~kW}(\# 12663)$ [34]. On the other hand, in the case of the inter-ELM profile with $T_{\mathrm{e} \_ \text {max }} \sim 70 \mathrm{eV}$ and $\lambda_{T e} \sim 0.6 \mathrm{~mm}$, the e-folding length is smaller than expected from intermachine scaling [2] of power decay length, because the exponential part of power profile should yield a smaller decay length than e-folding length (as the $j_{\text {sat }}$ decay length is also positive), which is contrary to the results of intermachine scaling for COMPASS plasma. It might be associated with a local minimum located around $R-R_{\mathrm{LCFS}} \sim 2$ $\mathrm{mm}$ as seen in Fig. 4. The distortion in the divertor inter-ELM $T_{\mathrm{e}}$ profile can be caused by SOL drift flows resulting from the large radial electric fields present in that region as shown in [35]. Note, when the fitted function [2] gives a larger $S$ parameter than the $\lambda$ parameter then the obtained value $\lambda$ is not necessarily correct. It is shown in [36] then the value $S$ needs to be smaller than $\lambda$ otherwise the fitted function [2] tends to provide too large $S$ and too low $\lambda$. However, the significant broadening of the ELM footprint in comparison with the inter-ELM were observed also during large Type-I ELMs on JET divertor [37, 38] and COMPASS outer midplane [7]. The new system of probes was used in an experiment devoted to detachment studies, which required monitoring of the electron temperature below $5 \mathrm{eV}$. As an example, the temporal evolution of the electron temperature near the outer strike point $(\sim 1 \mathrm{~cm})$ and the corresponding density ramp up during L-mode \#12748 $\left(I_{\mathrm{P}}=220 \mathrm{kA}, n_{\mathrm{e}}=4-9 \cdot 10^{19} \mathrm{~m}^{-3}\right)$ are shown in Fig. 5 . As the density was increased from 4 to $9 \cdot 10^{19} \mathrm{~m}^{-3}$, the electron temperature near the outer strike point decreased from $45 \mathrm{eV}$ to $\sim 1 \mathrm{eV}$, indicating development of plasma detachment in this region [32, 39, 40, 41]. Additionally, we have 
performed comparative measurements of the parallel heat flux, $q_{\|}$, using divertor probes and a fast infrared camera (IR) during L-mode discharge \#13043 $\left(I_{\mathrm{P}}=240 \mathrm{kA}, n_{\mathrm{e}}=4 \cdot 10^{19} \mathrm{~m}^{-3}\right)$, to benchmark the new array against another diagnostic system. The used camera was a Telops FAST-IR 2K equipped with a medium wavelength infrared (3-5 $\mu \mathrm{m}) \mathrm{InSb}$ detector and reaching a framerate of $4.4 \mathrm{kHz}$ with $192 \times 136 \mathrm{px}$. acquisition window. The camera was located at an outer midplane port and a mirror was used to observe a region in the outer divertor with a spatial resolution of $1 \mathrm{px} \sim 1.5 \mathrm{~mm}$ on the target plane. The target heat flux was calculated using the THEODOR code [42]. The incident parallel heat flux, $q_{\|}$, on divertor LPs is calculated using the formula $q_{\|}=\gamma \cdot T_{\mathrm{e}} \cdot I_{\mathrm{sat} \_\mathrm{i}} / S_{\mathrm{LP} \perp}$, where $\gamma$ is the heat power transmission coefficient for which we have used the value $\gamma=7$ assuming $T_{\mathrm{e}}=T_{\mathrm{i}}$ and negligible secondary electron emission [43]. The comparison between both $q_{\|}$profiles is shown in Fig. 6 . The data is mapped to the outer midplane and fitted by the "Eich function" [2] with excellent agreement of both diagnostics. We note that the same value $\gamma=7$ was applied for probe measurements in L-mode discharges also in other fusion experiments [17, $44,45,46]$. An extensive comparison of IR camera results with tile embedded Langmuir probe data was performed on the TCV tokamak to determine the heat power transmission coefficient in different L-mode discharges [47]. It was found that the empirical values of $\gamma$ are consistent within error bars with the expected theoretical values, neglecting particle reflection, secondary electron emission, surface recombination and assuming $T_{\mathrm{i}}=T_{\mathrm{e}}$.

We have further used the new probes to determine the ELM energy density $\varepsilon_{\|}$, which is calculated as the integral of

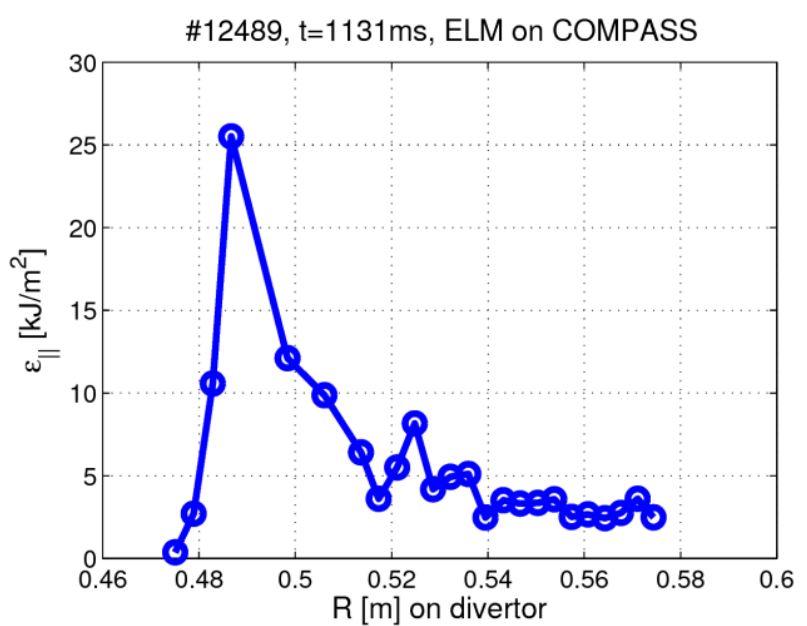

Fig. 7: Example of an ELM energy density profile on the LFS divertor target on COMPASS, $\varepsilon_{\|}$, from a single Type-I ELM with energy $\Delta \mathrm{W} / \mathrm{W} \sim 4.7 \%$, during NBI assisted H-mode discharge \#12489 $\left(P_{\mathrm{NBI}}=230 \mathrm{~kW}, I_{\mathrm{P}}\right.$ $=280 \mathrm{kA}, n_{\mathrm{e}}=6 \cdot 10^{19} \mathrm{~m}^{-3}$ ), as measured by the probe array. The peak value of $\varepsilon_{\|}$is $26 \mathrm{~kJ} / \mathrm{m}^{2}$.

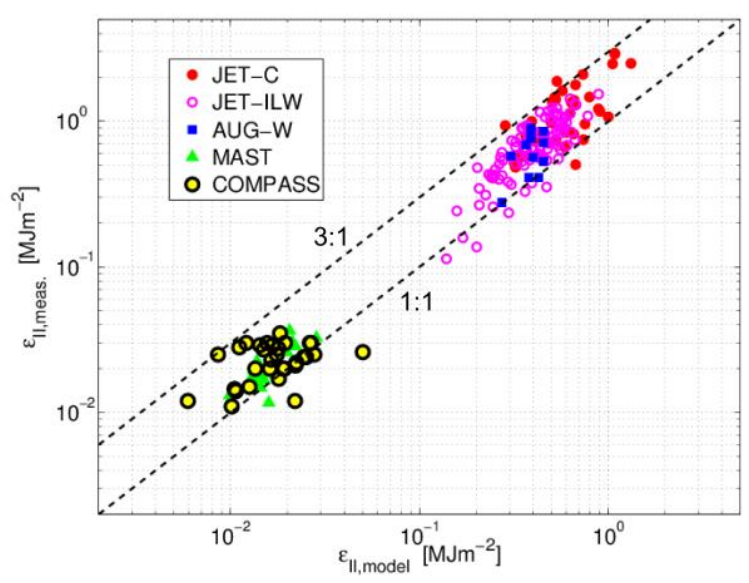

Fig. 8: Peak ELM energy density as measured by probes on the COMPASS outer divertor target (yellow circle) compared with values predicted by the model and results of IR measurements on JET, AUG, MAST [25]. The diagonal dashed line $1: 1$ or $3: 1$ represents the ratio between measured data and model. 
the parallel heat flux over the duration of an $\operatorname{ELM}\left(\Delta t_{\mathrm{ELM}}\right), \varepsilon_{\|}=\int_{\Delta t_{\mathrm{ELM}}} q_{\|}(t, R) \mathrm{dt}$. Indeed, the profile $q_{\|}$contains well resolved filamentary structure of each ELM, which is irregular. The ELM duration $\Delta t_{\mathrm{ELM}}$ is in the range of 100-200 mus. It is determined from spatially-integrated power on divertor LFS when it exceeds the inter-ELM value and then drops back down. As an example, the ELM energy density profile obtained during a Type-I ELM of relative energy $\Delta \mathrm{W} / \mathrm{W} \sim 4.7 \%$, during NBI assisted ELMy H-mode \#12489, is plotted in Fig. 7. The sample profile has a peak value $\max \left(\varepsilon_{\|}\right)=26 \mathrm{kJm}^{-2}$. To compute $q_{\|}$, we have used a heat power transmission coefficient $\gamma=7$, consistent with our Lmode measurements. We however admit that the collisional assumption, $T_{\mathrm{i}}=T_{\mathrm{e}}$, used to approximate the ion power flux may underestimate the ion power flux under low collisionality conditions [48]. The time dependence of the simulated heat power transmission coefficient of electrons and ions with respect to the evolution of $T_{i}$ and $T_{e}$ during a single ELM event is shown [49].

The peak of the ELM energy density, $\max \left(\varepsilon_{\|}\right)=26 \mathrm{kJm}^{-2}$, shown in Fig. 7 can be compared with the value predicted using the model [25]

$$
\varepsilon_{||}=\Delta_{\text {equi }} \cdot 2 \pi a_{\text {geo }} \sqrt{\frac{1+\kappa^{2}}{2}} \times \frac{3}{2} n_{\text {e,ped }} \cdot k_{B} \cdot T_{\text {e,ped }} \cdot \frac{B_{\text {tor }}}{B_{\text {pol }}}
$$

for the corresponding values of pedestal density $n_{\mathrm{e}, \mathrm{ped}}$ in $\left[10^{20} \mathrm{~m}^{-3}\right]$, temperature $T_{\mathrm{e}, \text { ped }}$ in $[\mathrm{keV}]$ and plasma elongation $\kappa$. The values $B_{\text {tor }}$ and $B_{\text {pol }}$ in [T] on the outer midplane as well as geometry factor $\Delta_{\text {equi }} \sim 1.5$ for COMPASS are obtained using the magnetic equilibrium reconstruction. In the case of the ELM event plotted in Fig. 7 ( $n_{\text {e,ped }}=3.75$ $\left.\cdot 10^{19} \mathrm{~m}^{-3}, T_{\mathrm{e}, \text { ped }}=250 \mathrm{eV}, B_{\text {tor }}=1.04 \mathrm{~T} B_{\text {pol }}=0.31 \mathrm{~T}, \kappa=1.81, a_{\text {geo }}=0.17 \mathrm{~m}\right)$ the model predicts $\max \left(\varepsilon_{\|}\right)=22 \mathrm{~kJ} \cdot \mathrm{m}^{-2}$ , which is in good agreement with our experimental observation. The systematic comparison of measured and predicted peak ELM energy density for different ELM events in several NBI assisted ELMy H-mode discharges is shown in Fig. 8. It can be seen that the experimental values are in a good agreement with the model, equation (2), in a range 1:1 up to 3:1, which is expected for different ELM energy [25]. We would like to stress that the experimental data from JET, AUG and MAST [25] are based solely upon conditionally-averaged IR camera measurements, while the COMPASS data can be obtained for a single ELMs. The low energy part of the model was so far only compared with data from MAST (which has a low aspect ratio), but the new COMPASS data shows that it is also applicable for an ITER-relevant shape. 


\section{CONCLUSION}

A new system of divertor Ball-pen probes and rooftop-shaped Langmuir probes was recently installed on the COMPASS tokamak to provide fast measurements of the plasma and floating potentials, the ion saturation current density, electron temperature and parallel heat flux. We have characterized the new Langmuir probes by systematic measurements of their $I-V$ characteristics and corresponding ratio of the electron and ion saturation currents $\Re=I_{\text {sat_e }}$ $/ I_{\text {sat_i. }}$. We have found a statistical value of $\Re=7.1 \pm 0.7$, which yields the coefficient $\alpha_{\mathrm{LP}}=2.0 \pm 0.1$. The electron temperature, $T_{\mathrm{e}}$, can then be calculated using the BPP and LP potentials as $T_{\mathrm{e}}=\left(\Phi^{\mathrm{BPP}}-V_{\mathrm{fl}}\right) / 1.4$. Using this technique, we have investigated electron temperature profiles during L-mode and ELMy H-mode discharges with well resolved ELM and inter-ELM values. A significant broadening of the e-folding length, $\lambda_{T e}$, was observed during Type-I ELMs. The maximum value at the divertor, $T_{\mathrm{e} \_ \text {max }} \sim 240 \mathrm{eV}$, was found comparable with the pedestal temperature $T_{\mathrm{e}, \text { ped }}$. The probes were also used to measure the electron temperature during detachment studies. The electron temperature near the outer strike point was observed to decrease to $\sim 1 \mathrm{eV}$ during L-mode discharges with density ramp-up, which we considered an indication for plasma detachment. We further performed first comparative measurements of the parallel heat flux using new divertor probes and a fast infrared camera during L-mode discharges on COMPASS. The resulting profiles showed excellent agreement between both techniques for a heat power transmission coefficient $\gamma=7$. Finally, ELM energy density, $\varepsilon_{\|}$, profiles were obtained during a set of NBI assisted ELMy H-mode discharges applying the same value of heat power transmission coefficient. The resulting peak values of $\varepsilon_{\|}$were compared with the predictions of the model [25]. We have found a good agreement between experimental and predicted values.

\section{ACKNOWLEDGMENTS}

This work was carried out within the EURATOM organization, and supported by the Czech Science Foundation project GA15-10723S and GA16-14228S and MSMT project \#LM2015045. It has been carried out within the framework of the EUROfusion Consortium and has received funding from the Euratom research and training programme 2014-2018 under grant agreement no. 633053. The views and opinions expressed herein do not necessarily reflect those of the European Commission. 


\section{REFERENCES}

1. W. Fundamenski et al., Nucl. Fusion 51 (2011) 083028 (19pp).

2. T. Eich et al., Nucl. Fusion 53 (2013) 093031 (7pp).

3. A. Kallenbach et al., Nucl. Fusion 48 (2008) 085008 (10pp).

4. J. P. Gunn et al., Nucl. Fusion 57 (2017) 046025 (35pp).

5. C. Silva et al., Plasma Phys. Control. Fusion 51 (2009) 105001.

6. H.W. Muller et al., Nucl. Fusion 51 (2011) 073023 (11pp).

7. J. Adamek et al., Nucl. Fusion 57 (2017) 022010.

8. J. G. Watkins et al., Rev. Sci. Instrum. 79, 10F125 (2008).

9. J. C. Xu et al., Rev. Sci. Instrum. 87, 083504 (2016).

10. G. F. Counsell et al., Plasma Phys. Control. Fusion 44 (2002) 827-843.

11. C. Silva et al., Contrib. Plasma Phys. 38 (1998) S, 47-52

12. V. Weinzettl et al., Fusion Eng. Des. 86 (2011) 1227-31.

13. Tsv. K. Popov et al., Plasma Sources Sci. Technol. 25 (2016) 033001 (18pp).

14. C. Guillemaut et al., Plasma Phys. Control. Fusion 57 (2015) 085006.

15. S. Jachmich et al., 36th EPS Conf. on Plasma Physics (Sofia Bulgaria, 29 June-3 July 2009), 2009, P-2.159 http:// epsppd.epfl.ch/Sofia/pdf/P2_159.pdf

16. D. Tskhakaya, S. Jachmich, T. Eich and W. Fundamenski, J . Nucl. Mater. 415 (2011) S860-4.

17. Jun-Gyo Bak et al., Fusion Engineering and Design 109-111 (2016) 836-842.

18. B. LaBombard and L. Lyons Rev. Sci. Instrum. 78 (2007) 073501.

19. J. Adamek et al., J. Nucl. Mater. 390-1 (2009) 1114-7.

20. J. Adamek et al., Contrib. Plasma Phys. 54, No. 3, 279 - 284 (2014).

21. C. Silva et al., Plasma Phys. Control. Fusion 57 (2015) 025003 (9pp).

22. J. Adamek et al., Rev. Sci. Instrum. 87 (2016) 043510.

23. N. R. Walkden et al., Rev. Sci. Instrum. 86 (2015) 023510.

24. S. Murphy-Sugrue et al., Plasma Phys. Control. Fusion 59 (2017) 055007 (7pp).

25. T. Eich et al. Proc. of the 22nd Int. Conf. on Plasma Surface Interactions in Controlled Fusion Devices (Rome, Italy 2016), J. Nucl. Mater. Energy, accepted (2017).

26. M. Weinlich, A. Carlson, Contrib. Plasma Phys. 36 (1996) S, 53-60.

27. J.P. Gunn, Phys. of Plasmas 4 (1997) 4435-4446.

28. G. F. Matthews, Plasma Physics and Controlled Fusion, Vol. 32, No. 14, pp. 1301 to 1320. (1990).

29. J. Horacek et al., Nucl. Fusion 50, 105001 (2010).

30. H. W. Müller et al., Contrib. Plasma Phys. 50, No. 9, 847 - 853 (2010).

31. D. Desideri and G. Serianni, Rev. Sci. Instrum. 69 (1998) 2354.

32. I. Duran et al., Journal of Nuclear Materials 463 (2015) 432-435.

33. R. Panek et al., Plasma Phys. Control. Fusion 58 (2016) 014015.

34. M. Komm et al., Nucl. Fusion 57 (2017) 056041 (7pp). 
35. C. G. Silva et al., Plasma Phys. Control. Fusion 40 (1998) 1159-1170.

36. T. Eich et al., Phys. Rev. Lett. 107 (2011) 215001.

37. T. Eich et al., Journal of Nuclear Materials 415 (2011) S856-S859.

38. H. Thomsen et al., Nucl. Fusion 51 (2011) 123001 (6pp).

39. A. Kallenbach et al., Nucl. Fusion 55 (2015) 053026 (8pp).

40. S. Potzel et al., Nucl. Fusion 54 (2014) 013001 (19pp).

41. T. D. Rognlien et al., Nuclear Materials and Energy (2017), in press, http://dx.doi.org/10.1016/j.nme.2016.12.002

42. A. Herrmann et al., Plasma Phys. and Control. Fusion 37(1995) 17.

43. P.C. Stangeby, Phys. Fluids 27 (1984) 682.

44. J. G. Watkins J.G. et al., J. Nucl. Mater. 390-1 (2009) 839-42.

45. G F Counsell et al., Plasma Phys. Control. Fusion 44 (2002) 827-843.

46. B. LaBombard at al., Phys. Plasmas 18 (2011) 056104.

47. J. Marki et al., Journal of Nuclear Materials 363-365 (2007) 382-388.

48. W. Fundamenski et al., Nucl. Fusion 45 (2005) 950-975.

49. R. A. Pitts et al., Nucl. Fusion 47 (2007) 1437-1448. 